\title{
Furthering our understanding of pathogen transmission in cystic fibrosis
}

\section{Lisa Saiman}

The source of pathogens for patients with cystic fibrosis (CF) is often unknown. Potential sources include the natural environment, the healthcare environment and other patients with CF. ${ }^{1}$ The relative contribution of these different sources is unknown, but the routes of transmission include direct and indirect contact with infected respiratory tract secretions and infectious respiratory droplets. ${ }^{12}$ Notably, airborne transmission - defined as dissemination of either airborne nuclei or small particles of respirable size-containing infectious agents (eg, Mycobacterium tuberculosis) and remaining infectious over time and distance has not been proved to be a route of transmission between patients with $\mathrm{CF}^{2}$

Infection control strategies to minimise the spread of potential pathogens are tailored to different modes of transmission. ${ }^{2}$ When caring for patients harbouring pathogens transmitted by contact with infectious secretions, staff perform hand hygiene before and after touching patients, patient care equipment or horizontal surfaces in patients' rooms and don gowns and gloves to prevent contamination of their clothing and hands. When caring for patients harbouring pathogens transmitted by infectious droplets, staff perform hand hygiene and don surgical masks. In contrast, when caring for patients infected by pathogens spread by airborne transmission, staff don an N95 respirator and patients are cared for in rooms with specific parameters for negative pressure, air exchanges and ventilation.

Infection control strategies to minimise transmission between patients with CF have been implemented in CF centres around the world. In addition to strategies outlined previously for staff, patients and their families are also taught to implement infection control practices including hand hygiene and respiratory hygiene which includes containment of respiratory tract

Correspondence to: Dr L Saiman, Division of Infectious Diseases, Department of Paediatrics College of Physicians and Surgeons of Columbia University, 633 West 168th Street, New York, NY10032, USA; Is5@columbia.edu secretions and maintaining a distance of at least $1 \mathrm{~m}$ from others with CF. ${ }^{1}$

Wainwright and colleagues ${ }^{3}$ have developed an experimental system to study the potential for airborne transmission of pathogens from patients with CF (see page 926). To do so, these investigators used a cough aerosol sampling method which included 5 min of voluntary coughing. Subjects coughed into a chamber and a vacuum pump pulled air-which included the particles generated during coughing-from the chamber through the stage impactors which contained 400 holes of decreasing diameter through which particles of relevant size penetrated and landed on agar plates for culture. Subjects included 28 adults and children with CF, 26 of whom had chronic infection with Pseudomonas aeruginosa, 13 of whom were studied during a pulmonary exacerbation and 18 of whom harboured the same strain, $P$ aeruginosa AES2.

$P$ aeruginosa was recovered from the subjects, the room air, on settle plates within the chamber and from the cough aerosols. However, as the authors are careful to state, their data do not prove the role of airborne transmission in CF. Their data demonstrate-via an experimental system with questionable relevance for clinical scenarios - that viable $P$ aeruginosa were collected on cough aerosol sampling plates and that $70 \%$ of generated particles were within the respirable range $(\leqslant 3.3 \mu \mathrm{m})$. However, their observations were potentially skewed by a dominant clone of transmissible $P$ aeruginosa whose behaviour may not be generalisable. Furthermore, the applicability of these findings to Gram-positive pathogens such as Staphylococcus aureus is unknown as these organisms were not studied.

Our understanding of droplet transmission is evolving thanks to improved studies of the epidemiology of viral pathogens such as rhinovirus, influenza and SARS. ${ }^{2}$ Historically, droplets have been defined as $>5 \mu \mathrm{m}$ in diameter with a defined risk distance of $\leqslant 1 \mathrm{~m}$ from the infected patient. However, we have learned that these parameters can vary depending on droplet characteristics and environmental conditions. Infectious droplet nuclei $\leqslant 5 \mu \mathrm{m}$ can arise from desiccation of a droplet, droplets $30 \mu \mathrm{m}$ in diameter can remain suspended in the air within a defined space such as a patient's room, and droplets can travel as far as $2-3 \mathrm{~m}^{2}$ Thus, Roy and Milton have proposed a new paradigm with which to understand the potential for airborne transmission. ${ }^{4}$ They proposed three categories for pathogens capable of airborne transmission: obligate (only transmitted by airborne transmission); preferential (can be transmitted by other routes such as droplet, but can also be transmitted by airborne transmission); and opportunistic (generally transmitted by other routes, but under special circumstances airborne transmission may occur).

While the relevance of this paradigm for CF is uncertain, it is important to keep an open mind regarding the potential for transmission of CF pathogens via the airborne route. Some CF centres have implemented the universal use of surgical masks by patients with CF to further reduce the risk of transmission and acquisition of potential pathogens. Use of masks has the further advantage of identifying CF patients to one another. Given the increasing importance of viral pathogens in CF and the influenza $A$ H1N1 (SO) pandemic, use of masks by patients with CF is prudent. However, mask use cannot replace other infection control practices such as hand hygiene and respiratory hygiene.

In summary, the current scientific understanding of the increased complexity of transmission of pathogens by the droplet and airborne route and the study presented by Wainwright and colleagues challenge our assumptions that we fully understand patient to patient transmission in CF. Additional studies are needed to expand the relevance of the observations by Wainwright and to demonstrate that actual transmission occurs via the airborne route in CF. Such studies would then allow us to modify our evidence-based infection control recommendations for CF.

Competing interests: None.

Provenance and peer review: Commissioned; not externally peer reviewed.

Thorax 2009;64:921-922. doi:10.1136/thx.2009.119495

\section{REFERENCES}

1. Saiman L, Siegel J, the CF Foundation Consensus Conference on Infection Control Participants. Infection control recommendations for patients with cystic fibrosis: microbiology, important pathogens, and infection control 
practices to prevent patient-to-patient transmission Infect Control Hosp Epidemiol 2003;24(Suppl 5):S1-52.

2. Siegel JD, Rhinehart E, Jackson M, et al, the Healthcare Infection Control Practices Advisory Committee. 2007 Guideline for Isolation Precautions. Preventing
Transmission of Infectious Agents in Healthcare Settings. http://www.cdc.gov/ncidod/dhqp/pdf/guidelines/ Isolation2007.pdf (accessed 18 Aug 2009).

3. Wainwright CE, France MW, O'Rourke P, et al.

Cough-generated aerosols of Pseudomonas aeruginosa and other Gram-negative bacteria from patients with cystic fibrosis. Thorax 2009;64:926-31.

4. Roy CJ, Milton DK. Airborne transmission of communicable infection - the elusive pathway. N Engl J Med 2004;350:1710-2

\section{Medication adherence in COPD: what have we learned?}

\section{MeiLan K Han}

As physicians, we take great interest in the adherence of our patients to prescribed medications. This is based on our belief that the benefits of treatment will be greater in patients who take their medication regularly and less among those who do not. C Everett Koop is quoted as having said: "Drugs don't work in patients who don't take them". The practice of faithfully complying with a medication regimen, however, may be just as important as the efficacy of the medication itself. In this month's issue of Thorax, Vestbo et al ${ }^{1}$ report that, among patients with chronic obstructive pulmonary disease (COPD) participating in the TORCH trial, ${ }^{2}$ adherers experienced significantly better survival and a lower risk of hospital admission due to exacerbations than non-adherers (see page 939). These effects, however, were independent of treatment group.

In fact, a growing body of literature suggests that adherence to drug therapy in clinical trials-both medication and placebo regimens-predicts better outcomes. Many of these data have been generated from cardiovascular trials. ${ }^{3}$ In 1980 a report from the Coronary Drug Project significantly challenged traditional scientific thought regarding the relationship between adherence and disease outcomes. $^{4}$ This randomised double-blind placebo controlled trial examined the effects of clofibrate on the survival of men who had experienced a myocardial infarction. ${ }^{5}$ Five-year mortality was similar for those treated with medication $(20 \%)$ and those treated with placebo (21\%). When analysed by adherence patterns, however, good adherers (defined as those taking at least $80 \%$ of the prescribed medication) had significantly lower 5-year mortality than poor adherers (15\% vs 25\%). Surprisingly, however, these findings were nearly identical in the

Correspondence to: $\mathrm{Dr} \mathrm{M} \mathrm{K}$ Han, University of Michigan, 1500 E Medical Center Drive, 3916 TC, Ann Arbor, Ml 48109-5360, USA; mrking@umich.edu placebo group (15\% vs $28 \%$ mortality). Initially, much discussion focused on the potential for bias in post hoc analyses. As more data have accumulated, however, the scientific community has begun to realise that, across disciplines and treatment assignments, adherent behaviour in clinical trials is associated with better outcomes. ${ }^{3}$

While it has been hypothesised that improved outcomes for adherers may be due to selection bias with good adherers having less severe disease, this is probably not the case. ${ }^{4}$ The improved outcome for adherers in the study by Vestbo et al remained after adjustment for disease severity as measured by forced expiratory volume in $1 \mathrm{~s}$ and dyspnoea measured by the MRC dyspnoea scale. ${ }^{1}$ Similar results have been reported in other trials. ${ }^{4}$ In fact, the Lung Health Study reported that better adherence was associated with more severe airways obstruction. ${ }^{6}$ Another hypothesis is that adherence to drug treatment may reflect a patient's biological, social and psychological makeup which may all influence outcomes. Adherence may be a surrogate marker for other healthy behaviours such as taking medications for comorbid conditions, eating more healthily or taking safety precautions such as wearing seatbelts or sunscreen. Alternatively, however, the adherence itself and the expectation of treatment effect may activate behaviours that are then partly responsible for improved outcomes. ${ }^{7}$ For instance, the act of adhering to a treatment regimen may instil a sense of well-being and reduce anxiety about a chronic disease or may lead to altered health habits such as exercise or smoking cessation.

Very few data exist regarding adherence to inhaled medication in COPD. It was previously reported that only about 37\% of patients with chronic lung disease are fully adherent with medical treatments in general. ${ }^{8}$ Long-term adherence with inhaled medications was reported from the Lung Health Study. ${ }^{6}$ Self-reported data suggested that nearly $70 \%$ of patients adhered to the medication regimen, dropping off slightly over the next 18 months. Adherence confirmed by canister weights, however, showed that only $48 \%$ of patients had good adherence at 1 year. Two other studies of nebulised treatments in COPD suggest that only about half of patients take their medications regularly. ${ }^{9}{ }^{10}$ In the current study, $80 \%$ of subjects demonstrated good adherence (defined as adherence to study medications of $>80 \%$ over the entire period the subject was in the study). While this is a good percentage within the context of a clinical trial, it probably does not reflect "real life" patient behaviour.

One would hope that the patients who need medication most might also be the group that is most adherent. It is therefore disappointing that, in the study by Vestbo et $a l,{ }^{1}$ no association was found between disease severity defined by GOLD stage and adherence. In fact, poor adherers actually had higher MRC dyspnoea scores. However, it should also be noted that adherence in this study was determined by the percentage of returned drug left unused. What this analysis does not take into account is the significantly greater number of patients who dropped out of the placebo arm compared with the combination therapy arm $(44.2 \%$ vs $34.1 \%$ ), which also could be interpreted as non-adherence in the broadest sense of the word. These data suggest that adherence in this more global sense is probably less than the reported $80 \%$. They also suggest that symptomatic improvement or drug efficacy may have had an impact on study participation even if the analysis of medication adherence among those who stayed in the trial did not differ between treatment and placebo arms.

In summary, this study indicates that adherence to inhaled therapy in COPD within the context of a clinical trial is good and associated with improved outcomes. Ultimately, however, if this is due to qualities intrinsic to the adherer, this actually leaves little room for the physician to modify outcomes other than to prescribe the treatment itself. If, however, the act of adhering actually activates other positive behaviours, then we as physicians should be even more aggressive about creating structured treatment 